\title{
A method to search for topological signatures in the angular distribution of cosmic objects
}

\author{
A. Bernui ${ }^{\star}$ and T. Villela
}

Instituto Nacional de Pesquisas Espaciais, Divisão de Astrofísica, Av. dos Astronautas 1758, 12227-010, São José dos Campos, SP, Brazil

e-mail: [bernui; thyrso]@das.inpe.br

Received 5 September 2004 / Accepted 30 September 2004

\section{ABSTRACT}

We present a method to search for large angular-scale correlations, termed topological signatures, in the angular distribution of cosmic objects, which does not depend on cosmological models or parameters and is based only on the angular coordinates of the objects. In order to explore Cosmic Microwave Background temperature fluctuations data, we applied this method to simulated distributions of objects in thin spherical shells located in three different multiply-connected Euclidean 3-spaces $\left(T^{3}, T_{\pi}\right.$, and $\left.G_{6}\right)$, and found that the topological signatures due to these topologies can be revealed even if their intensities are small. We show how to detect such signatures for the cases of full-sky and partial-sky distributions of objects. This method can also be applied to other ensembles of cosmic objects, like galaxies or quasars, in order to reveal possible angular-scale correlations in their distributions.

Key words. cosmology - cosmology: large-scale structure of Universe - cosmology: cosmic microwave background - methods: data analysis

\section{Introduction}

Homogeneity property refers to the spatial distribution of cosmic objects in the Universe, and to test it one needs to know the angular positions of these objects in the celestial sphere and their distances to us as well. Unfortunately, cosmological distances are not directly measured, as they are calculated through the redshift-distance relationship, which involves cosmological parameters still not known with enough accuracy. So, precise distances to extragalactic objects are not currently available. Isotropy, instead, deals with the angular distribution of objects in the sky. Then, only two angular coordinates for each object are needed to determine whether or not they are isotropically distributed around us. Nowadays such coordinates are accurately measured and there are available catalogs for several types of objects, like quasars, gamma-ray bursts, and the (sky pixels corresponding to the) Cosmic Microwave Background Radiation (CMBR) temperature fluctuations. In analyzing such catalogs, some questions arise: How can we recognize whether a given class of cosmic objects follows an exact or a quasiexact isotropic distribution? How can we quantify small deviations from a purely isotropic distribution? How can we distinguish a possible cosmological pattern or topological signature imprinted in these catalogs from pure statistical fluctuations?

The study of the large-scale (i.e. global) homogeneity and isotropy of the Universe encompasses its topology as well, since topological properties are global properties of 3-spaces.

* On leave from Universidad Nacional de Ingeniería, Facultad de Ciencias, Apartado 31 - 139, Lima 31, Peru.
Multiply-connectedness is a topological property that tesselates a simply-connected space (like the Euclidean space $\mathcal{R}^{3}$ ) producing multiple images of a given object. This property generates distance and angular correlations (i.e. anisotropies) in the distribution of objects. The Small Universe hypothesis (Ellis \& Schreiber 1986), which assumes that the Universe is a compact (i.e. multiply-connected with finite volume) 3-space that lies inside - at least partially - the Last Scattering Surface (LSS), has not been discarded by recent CMBR data. In view of this situation, we are motivated to study the anisotropic signatures of topological origin in the angular distribution of cosmic objects, and for this we consider simulated ensembles of objects in different Euclidean multiply-connected 3-spaces.

In order to search for these signatures in astronomical data sets, we developed a method based on histograms of angular separations between pairs of cosmic objects. We call it the Pair Angular Separation Histogram (PASH) method. We are particularly interested in studying angular correlations in simulated catalogs of cosmic objects that share the same attributes as the photons of the CMBR temperature fluctuations, i.e., point-like objects located in the (comoving 3-dimensional) thin shell representing the decoupling era, but viewed from Earth as located on the celestial sphere. As we shall show, the correlations originated by the isometry properties of the compact 3-spaces can be revealed by the method we propose here. This method resembles the Cosmic Crystallography (CC) method (Lachièze-Rey \& Luminet 1995; Lehoucq et al. 1996; Fagundes \& Gausmann 1998a,b; Gomero et al. 2000a,b, 2001, 2002). However, there is a basic difference between them in that here we just look 
for angular correlations instead of searching for distance correlations as the $\mathrm{CC}$ method does. Our method is therefore independent of any cosmological model or parameters, while the CC method depends on this information to calculate the radial distance of cosmic objects from their measured redshifts.

In Sect. 2 we present the basic properties of the 3-spaces representing our Universe in the Friedmann-Lemaître cosmological models (Friedmann 1922; Lemaître 1927). In Sect. 3, we present the geometrical and statistical method we developed to reveal correlations between pairs of objects distributed in a 2-dimensional spherical surface. The treatment of this problem initiates with the theoretical description of a perfectly isotropic distribution of objects in a spherical surface $\mathcal{S}^{2}$. For this type of data we construct the normalized expected probability density for two objects be separated by a given angle, separation that of course ranges in the interval $[0, \pi]$. This probability gives rise to the Expected Pair Angular Separation Histogram (EPASH) ${ }^{1}$. We also present the EPASH for partial-sky catalogs containing objects distributed only in polar-caps. In Sect. 4, we present the theoretical deduction of the topological signatures due to pure translational isometries, also known as Clifford translations, appearing in the PASHs. We illustrate these results, in Sect. 5, with numerical simulations using catalogs that contain multiple images of cosmic objects generated by the isometries of the following Euclidean compact (i.e. multiply-connected with finite volume) orientable 3-spaces: $T^{3}, T_{\pi}$, and $G_{6}$ (denoted by $\mathcal{G}_{1}$, $\mathcal{G}_{2}$, and $\mathcal{G}_{6}$, respectively, in Wolf's classification Wolf 1984). In this section we also show that performing the mean of several PASHs (or Mean Pair Angular Separation Histogram MPASH) reduces significantly the statistical noise, which allows us to reveal the anisotropies due to a non-translational isometry whose tiny signature is present in the angular distribution of cosmic objects. We analized this problem considering MPASHs for full-sky catalogs as well as for polar-cap catalogs. Finally, in Sect. 6, we discuss our results and also the problem of finding suitable catalogs of cosmic objects from the available astronomical data.

\section{The geometry of the 3-spaces}

The manifold $T^{3}$, best known as the three-torus, has its Fundamental Polyhedron (FP) usually -but not necesarily- represented by an equal-sided cube, where its three pairs of faces are identified by pure (or Clifford) translation isometries. The manifold $T_{\pi}$ has two pairs of faces identified by pure translation isometries and one pair of faces identified after a rotation of $180^{\circ}$, the so-called screw-motion isometry. The manifold $G_{6}$ has no pair of faces of its FP identified by a pure translation $^{2}$. In analyzing the distributions of objects in these 3 -spaces we shall be considering the effect of not only pure translational isometries but also translation-with-rotation isometry, or screw-motion. For completeness, we find interesting to

\footnotetext{
${ }^{1}$ This result is independent of the three-dimensional geometry since all three isotropic geometries (i.e. spherical $\mathcal{S}^{3}$, Euclidean $\mathcal{R}^{3}$, and hyperbolic $\mathcal{H}^{3}$ ) possess spherical surfaces $\mathcal{S}^{2}$ as hypersurfaces.

2 A picture of this FP can be seen, for instance, in Bernui et al. (1998), where it is denoted by $\mathcal{T}_{4}$.
}

briefly review here the basic properties of compact 3-spaces of constant curvature.

The large-scale structure of the Universe is described by the Friedmann-Lemaître cosmological models. In these models, the local geometry satisfies the local homogeneity and isotropy properties and is described by the Robertson-Walker (RW) line element $\mathrm{d} s^{2}=\mathrm{d} t^{2}-a^{2}(t) \mathrm{d} \sigma^{2}$, where $a(t)$ is the scale factor, and $\mathrm{d} \sigma^{2}=\mathrm{d} \chi^{2}+F_{k}^{2}(\chi)\left[\mathrm{d} \theta^{2}+\sin ^{2} \theta \mathrm{d} \varphi^{2}\right]$ is the line element of the $t=$ constant spatial sections. The functions $F_{k}(\chi) \equiv \sin \chi, \chi, \sinh \chi$, where $k$ is the normalized curvature parameter, which can take the values $+1,0,-1$, corresponding to the spherical, Euclidean, and hyperbolic geometry, respectively. It is known that the three-dimensional spatial sections (hereafter denoted by $\mathcal{M}_{k}$ ) are manifolds of constant curvature obtained from the quotient $\mathcal{M}_{k}=\widetilde{\mathcal{M}}_{k} / \Gamma$ (see e.g. Wolf 1984), where $\Gamma$ is a discrete group of isometries of the manifold $\widetilde{\mathcal{M}}_{k}$, without fixed points. The manifold $\widetilde{\mathcal{M}}_{k}$ is termed the universal covering of $\mathcal{M}_{k}$. Therefore, while $\widetilde{\mathcal{M}}_{k}$ is a simply-connected manifold, $\mathcal{M}_{k}$ is a multiply-connected one. We would like to stress that the values $k=+1,0,-1$ in the RW line element determines only the local geometry of $\mathcal{M}_{k}$ (equivalently that of $\widetilde{\mathcal{M}}_{k}$ ).

Since the simply-connected $\widetilde{\mathcal{M}}_{k}$ and the multiplyconnected $\mathcal{M}_{k}$ manifolds share the assumed local homogeneity and isotropy properties, we conclude that both manifolds are good solutions of the RW local geometry, i.e., the EinsteinHilbert equations. Consequently, General Relativity (or any other metrical theory) can not distinguish between $\widetilde{\mathcal{M}}_{k}$ and $\mathcal{M}_{k}$ because they look like each other locally.

In a Universe with a multiply-connected 3-space $\mathcal{M}_{k}$, and whenever the horizon scale is greater than half of the smallest closed geodesic of $\mathcal{M}_{k}$, multiple images of a given cosmic object might exist due to the isometries of the group $\Gamma^{3}$. These images originate distance correlations between the mapped cosmic objects and also produce angular correlations between those distance-correlated objects. As we shall see, these angular correlations can be revealed by the PASH method described in the next section.

\section{PASHs}

\subsection{EPASHs for full-sky catalogs}

In essence, a PASH is a normalized plot of the number of pairs of objects versus the angular distance between them. A formal construction of a PASH is as follows: let $\mathcal{B}_{R} \subset \mathcal{M}_{k}$ be a ball of radius $R$ centered at the origin of coordinates $O$, and involving spherical surface $\mathcal{S}_{R}$, and containing an ensemble of (cosmic) objects. We call $\mathcal{B}_{R}$ the observable Universe, $O$ represents the Earth, $R_{-}$is the radius of the LSS, $\mathcal{S}_{R}$ is the celestial sphere with radius $R \equiv R_{+}=R_{-}+\Delta R$, where $\Delta R$ is the thickness of the decoupling era. Since we are interested in analyzing CMB data, our simulations consider ensembles of objects located in a thin shell of thickness $\Delta R$, which are after projected onto the celestial sphere $\mathcal{S}_{R}$ ignoring their radial coordinate. However, we

\footnotetext{
${ }^{3}$ Except when $\Gamma=$ Identity, in which case $\mathcal{M}_{k}=\widetilde{\mathcal{M}}_{k}$ is a simplyconnected manifold.
} 
point out that this method can be applied to any spatial distribution of cosmic objects. Given a set of well-defined selection rules $^{4}$, a catalog $C$ is a list of all objects in $\mathcal{B}_{R}$ which satisfies a sub-set of the whole set of rules.

We start the description of the method by firstly showing how to obtain the PASH. Let's assume that we have an ensemble of objects that are, by assumption, isotropically distributed in $\mathcal{S}_{R}$ (see Eq. (5)). A full-sky map catalog $C$ is a list of $N$ objects spreaded out in $\mathcal{S}_{R}$ with their corresponding angular coordinates. We divide the interval $(0, \pi]$ in $m$ bins of equal length $\delta \gamma=\pi / m$, where each sub-interval has the form $J_{i}=\left(\gamma_{i}-\frac{\delta \gamma}{2}\right.$, $\left.\gamma_{i}+\frac{\delta \gamma}{2}\right], i=1,2, \ldots, m$, with center in $\gamma_{i}=\left(i-\frac{1}{2}\right) \delta \gamma$. Now, we denote by $\phi(\gamma)$ the number of pairs of objects in $C$ separated by a distance $\gamma \in(0, \pi]$. Thus,

$\Phi\left(\gamma_{i}\right)=\frac{2}{N(N-1) \delta \gamma} \sum_{\gamma \in J_{i}} \phi(\gamma)$

is the normalized counting of the number of pairs of objects, separated by an angle $\gamma_{i}$, that lies in the sub-interval $J_{i}$. Actually, $\sum_{i=1}^{m} \Phi\left(\gamma_{i}\right) \delta \gamma=1$, so we can average several functions $\Phi\left(\gamma_{i}\right)$, each one built from catalogs having a comparable number of objects.

It may occur that some angular correlations of small intensity are present in a given PASH, but are not clearly seen. To reveal them, and since PASH and EPASH are normalized, we subtract the PASH from the EPASH in order to enhance possible angular correlations.

Let us now obtain the EPASH for the simply-connected manifold $\widetilde{\mathcal{M}}_{k}$. Let $p, q \in \mathcal{B}_{R} \subset \widetilde{\mathcal{M}}_{k}$ be an arbitrary pair of objects listed in $C$ with coordinates $\left(r_{p}, \theta_{p}, \varphi_{p}\right),\left(r_{q}, \theta_{q}, \varphi_{q}\right)$, respectively. Then, the probability density that two completely uncorrelated objects ${ }^{5} p$ and $q$ be separated by an angle $\gamma \in$ $(0, \pi]$, is

$\mathcal{P}(\gamma)=\int_{\mathcal{B}_{R}} \int_{\mathcal{B}_{R}} \mathrm{~d}^{3} r_{p} \mathrm{~d}^{3} r_{q} \rho\left(\bar{r}_{p}\right) \rho\left(\bar{r}_{q}\right) \delta(\Lambda(p, q)-\gamma)$,

where $\bar{r}_{p}, \bar{r}_{q}$ are the vectors from $O$ to the position of the objects $p$ and $q$, respectively. In $\widetilde{\mathcal{M}}_{0}=\mathcal{R}^{3}$, the angular separation between $p$ and $q$ is given by

$\Lambda(p, q) \equiv \arccos \left[\cos \theta_{p} \cos \theta_{q}+\sin \theta_{p} \sin \theta_{q} \cos \left(\varphi_{p}-\varphi_{q}\right)\right]$,

where $\theta_{p}, \theta_{q} \in[0, \pi]$ and $\varphi_{p}, \varphi_{q} \in[0,2 \pi]$. Note that $\rho\left(\bar{r}_{p}\right) d^{3} r_{p}$ is the probability of the object $p$ to be in the volume $d^{3} r_{p}$. The probability of finding $p$ in the whole $\mathcal{B}_{R}$ should of course be equal to 1 , i.e.,

$\int_{\mathcal{B}_{R}} \rho\left(\bar{r}_{p}\right) \mathrm{d}^{3} r_{p}=1$.

Thus, a purely isotropic and normalized density distribution of objects observed in $\mathcal{S}_{R}$ reads

$\rho\left(\bar{r}_{p}\right)=\frac{\delta\left(r_{p}-R\right)}{4 \pi R^{2}}$.

\footnotetext{
${ }^{4}$ i.e. physical properties that are common to the cosmic objects in a given catalog, such as luminosity threshold, redshift, etc.

5 This situation corresponds to the monopole term $(l=0)$ in a multipole decomposition.
}

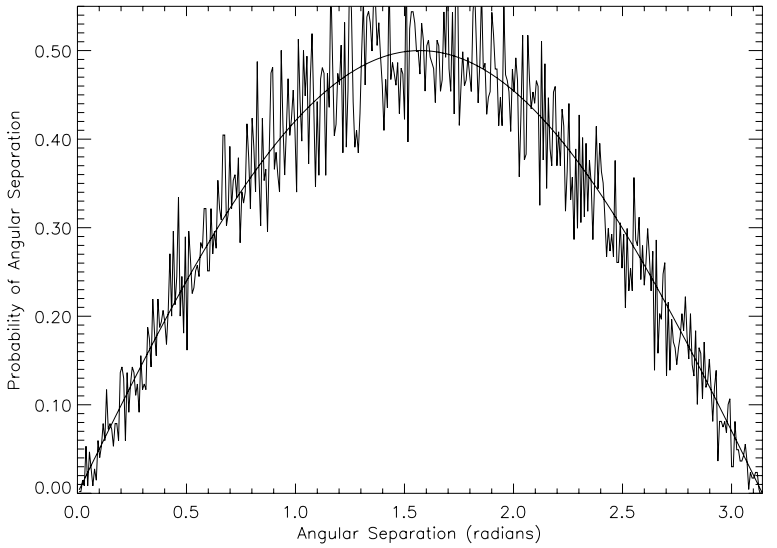

Fig. 1. EPASH (smooth curve) and PASH (noisy curve) for a full-sky simulated catalog with $N=200$ objects in $\mathcal{S}_{R} ; m=400$ bins.

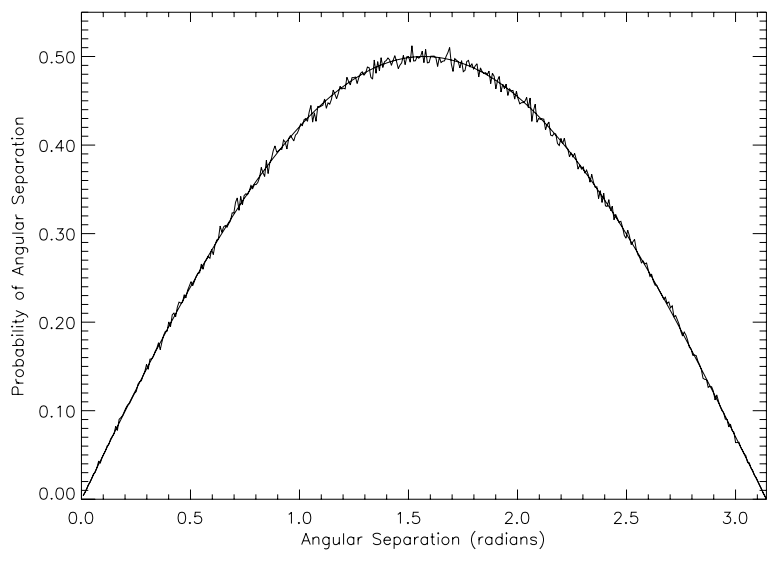

Fig. 2. EPASH (smooth curve) and PASH (noisy curve) for a full-sky simulated catalog with $N=2 \times 10^{3}$ objects in $\mathcal{S}_{R} ; m=400$ bins.

Finally, integrating (2) we obtain

$\mathcal{P}(\gamma)=\frac{1}{2} \sin \gamma$

Hence, the EPASH is simply defined by

$\Phi_{\text {expected }}\left(\gamma_{i}\right) \equiv \frac{1}{\delta \gamma} \int_{J_{i}} \mathcal{P}(\gamma) \mathrm{d} \gamma$

However, if the interval $J_{i}$ is small enough (as shall be considered here), a suitable approximation for the EPASH is

$\Phi_{\text {expected }}\left(\gamma_{i}\right) \simeq \mathcal{P}\left(\gamma_{i}\right)$.

We also note that $\mathcal{P}(\gamma)$ defined in Eq. (2) satisfies the normalization property, $\int_{0}^{\pi} \mathcal{P}(\gamma) \mathrm{d} \gamma=1$, which is a useful condition since it let us to perform the mean of an arbitrary number of histograms obtained with catalogs containing a comparable number of objects.

In Figs. 1 and 2 we show the EPASH together with the corresponding PASHs obtained from numerical simulations for full-sky catalogs with $N=200$ and $N=2 \times 10^{3}$ objects, respectively.

\subsection{EPASHs for polar-cap catalogs}

Our galaxy poses a serious problem to the use of full-sky CMBR maps and for other sky catalogs as well, because it 
contaminates part of the celestial sphere around the Galactic plane. This fact makes somewhat useless the approach we developed studying the angular distribution of cosmic objects listed in full-sky catalogs. For this reason, we also studied the PASHs for catalogs containing objects located in polar-caps (i.e. above or below the equator of a spherical distribution).

Consider a catalog with objects located in a spherical cap surface $S_{\mathrm{PC}} \subset \mathcal{S}^{2}$, centered, for instance, around the $z$-axis, which is assumed to be aligned with the North Pole. We call this ensemble a polar-cap catalog, $C_{\mathrm{PC}}$. In this case, any object with coordinates

$$
\begin{aligned}
& x=R \cos \varphi \sin \theta, \\
& y=R \sin \varphi \sin \theta, \\
& z=R \cos \theta,
\end{aligned}
$$

where $\varphi \in[0,2 \pi]$ and the azimuthal angle $\theta \in\left[0, \theta_{0}\right]$, with $\theta_{0} \leq$ $\pi / 2$, is in the surface $S_{\text {PC }}$. Thus, the isotropic and normalized density distribution of objects in $S_{\mathrm{PC}}$ is

$\rho\left(\bar{r}_{p}\right)=\frac{\Theta\left(\theta_{0}-\theta_{p}\right) \delta\left(r_{p}-R\right)}{A_{\mathrm{PC}}}$,

where $A_{\mathrm{PC}} \equiv 2 \pi R^{2}\left(1-\cos \theta_{0}\right)$ is the area of the polar-cap. Using this information in the definition of an EPASH (Eq. (2)) and after the integration of the $\delta$ Dirac-function, we obtain

$\mathcal{P}_{\mathrm{PC}}\left(\gamma ; \theta_{0}\right)=\frac{R^{4}}{A_{\mathrm{PC}}^{2}} \int_{0}^{\theta_{0}} \sin \theta_{p} \mathrm{~d} \theta_{p} \int_{0}^{\theta_{0}} \sin \theta_{q} \mathrm{~d} \theta_{q} I\left(\theta_{p}, \theta_{q} ; \gamma\right)$,

where

$I\left(\theta_{p}, \theta_{q} ; \gamma\right) \equiv \int_{0}^{2 \pi} \int_{0}^{2 \pi} \mathrm{d} \varphi_{p} \mathrm{~d} \varphi_{q} \delta(\Lambda(p, q)-\gamma)$.

It can be shown that this integral is equal to

$I\left(\theta_{p}, \theta_{q} ; \gamma\right)=\frac{4 \pi \sin \gamma}{\sin \theta_{p} \sin \theta_{q} \sin \alpha\left(\theta_{p}, \theta_{q} ; \gamma\right)}$,

where the function $\alpha$ is defined by

$\alpha\left(\theta_{p}, \theta_{q} ; \gamma\right) \equiv \arccos \left[\frac{\cos \gamma-\cos \theta_{p} \cos \theta_{q}}{\sin \theta_{p} \sin \theta_{q}}\right]$

and lies in the interval $[0, \pi]$. Due to this condition, $\theta_{p}$ and $\theta_{q}$ should satisfy the relation

$\frac{\cos \gamma-\cos \theta_{p} \cos \theta_{q}}{\sin \theta_{p} \sin \theta_{q}} \in[-1,1]$.

Equivalently, this constraint for the values of $\theta_{p}$ and $\theta_{q}$ can be written as the following two inequalities:

$\theta_{p}+\theta_{q} \geq \gamma$

$\left|\theta_{p}-\theta_{q}\right| \leq \min \left\{\theta_{0}, \gamma\right\}$

Thus, the probability density to observe two objects in a polar cap (defined by the angle $\theta_{0}$ ) with an angular separation $\gamma$ is

$\mathcal{P}_{\mathrm{PC}}\left(\gamma ; \theta_{0}\right)=\frac{4 \pi R^{4} \sin \gamma}{A_{\mathrm{PC}}^{2}} \int_{0}^{\theta_{0}} \mathrm{~d} \theta_{p} \int_{0}^{\theta_{0}} \frac{\mathrm{d} \theta_{q}}{\sin \alpha\left(\theta_{p}, \theta_{q} ; \gamma\right)}$,

with the conditions given in Eq. (16). The double integral in Eq. (17) can be numerically solved, but it is convenient to do

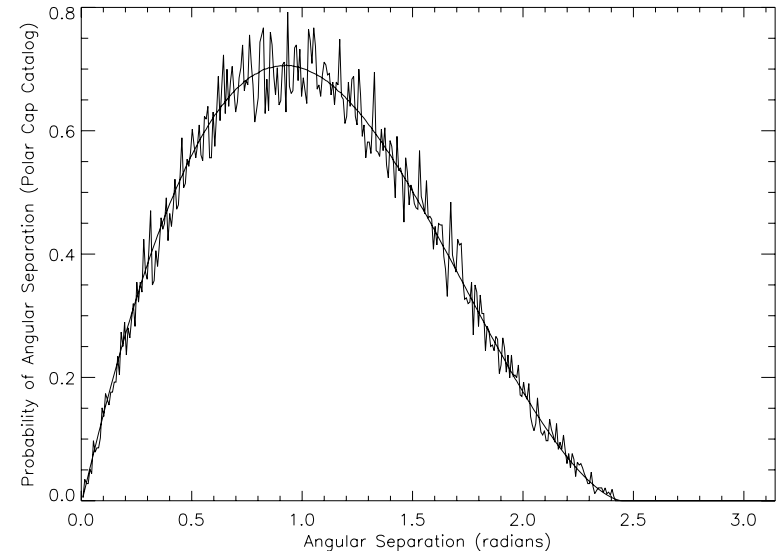

Fig. 3. EPASHpc (smooth curve) and PASH (noisy curve) for a polarcap simulated catalog with $\theta_{0}=70^{\circ}, N_{\mathrm{PC}}=332$ objects $\left(10^{3}\right.$ objects in the full-sky simulated catalog, $m=400$ bins); $\sigma=0.02799$.

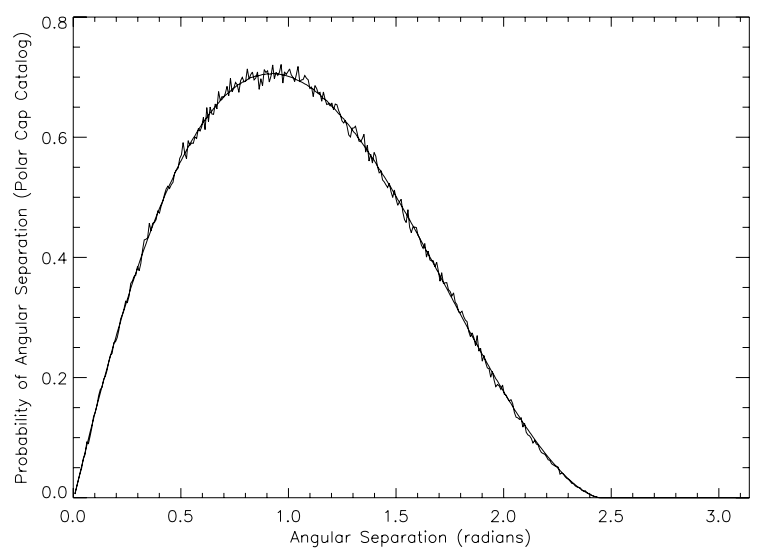

Fig. 4. EPASHpc (smooth curve) and PASH (noisy curve) for a polarcap simulated catalog with $\theta_{0}=70^{\circ}, N_{\mathrm{PC}}=1337$ objects (with $4 \times 10^{3}$ objects in the full-sky simulated catalog, $m=400$ bins); $\sigma=0.00661$.

it by parts considering carefully the conditions imposed by the inequalities in Eq. (16). Thus, given $\theta_{0}$, we can obtain the probability density $\mathcal{P}_{\mathrm{PC}}$ as a function of the angular separation $\gamma$.

Recently, the analytical expression for the expected probability density that an arbritary pair of objects in $S_{\text {PC }}$ be separated by $\gamma$ degrees, i.e. EPASH for polar-caps (EPASHpc), has been found by Teixeira (2003), who obtained

$$
\begin{aligned}
\mathcal{P}_{\mathrm{PC}}\left(\gamma ; \theta_{0}\right)=\frac{4 \pi R^{4} \sin \gamma}{A_{\mathrm{PC}}^{2}}\left(\operatorname { a r c s i n } \left[\left\{\left(\cos \theta_{0}+\cos \gamma\right)\right.\right.\right. \\
\left.\left.\quad \times \sqrt{\cos \gamma-\cos 2 \theta_{0}}\right\} /\left\{\sin \theta_{0}\left(1+\cos \theta_{0}\right) \sqrt{1+\cos \gamma}\right\}\right] \\
\left.\quad+\left(1-2 \cos \theta_{0}\right) \arccos \left[\cot \theta_{0} \tan (\gamma / 2)\right]\right) .
\end{aligned}
$$

To illustrate this result, we simulate two full-sky catalogs with $N=10^{3}$ and $N=4 \times 10^{3}$ objects, respectively. We show in Figs. 3 and 4 the EPASHpc together with the corresponding PASHs obtained from these simulations, where the number of objects in the polar-caps were $N_{\mathrm{PC}}=332$ and $N_{\mathrm{PC}}=1337$, respectively. 


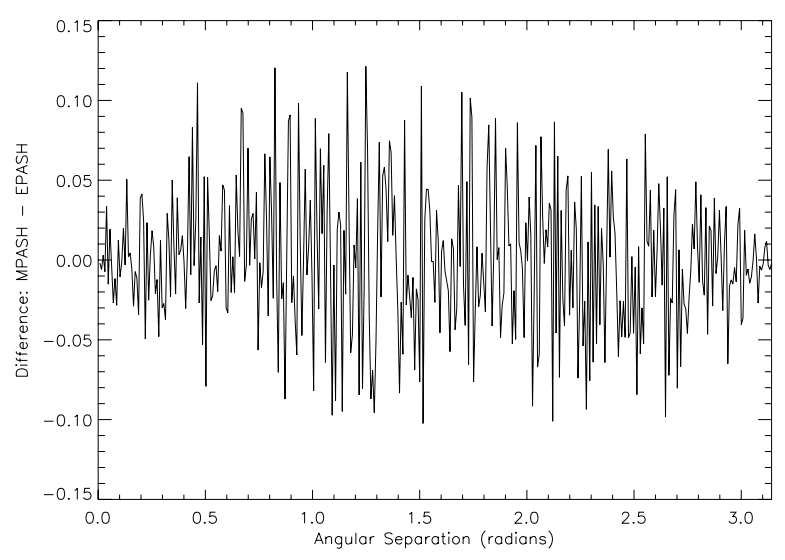

Fig. 5. Difference between PASH and EPASH plotted in Fig. 1 for a full-sky simulated catalog $(K=1)$ with $N=200$ objects and $m=$ 400 bins; $\sigma=0.04418$.

\subsection{MPASHs for full-sky and polar-cap catalogs}

Suppose that $K$ comparable catalogs $C_{k}, k=1,2, \ldots, K$, of objects located in the celestial sphere are available for analysis. For a given $m$ (common to all the PASHs), we calculate the functions

$\Phi_{k}\left(\gamma_{i}\right)=\frac{2}{N_{k}\left(N_{k}-1\right) \delta \gamma} \sum_{\gamma \in J_{i}} \phi_{k}(\gamma)$,

where $N_{k}$ is the number of objects in $C_{k}$, and $\phi_{k}(\gamma)$ is the number of pairs of objects in $C_{k}$ with angular separation $\gamma$. Then, the MPASH is defined by

$\left\langle\Phi\left(\gamma_{i}\right)\right\rangle \equiv \frac{1}{K} \sum_{k=1}^{K} \Phi_{k}\left(\gamma_{i}\right)$.

Indeed, we know that performing the average of Pair Separation Histograms in the CC method, the result is that the statistical fluctuations are reduced by a factor proportional to $1 / \sqrt{K}$ but the topological information is preserved (Gomero et al. 2000a,b, 2001). In the next section we shall see that for the PASHs a similar situation occurs, that is, the greater the number of PASHs considered in the average, the better the approximation of the MPASH to the corresponding EPASH.

\subsection{Noise in PASHs}

In Figs. 5 and 6 we show the difference between the PASH and the EPASH, already plotted in Figs. 1 and 2, respectively. The noise of a PASH, as observed in Figs. 5 and 6, is simply given by

$\eta\left(\gamma_{i}\right)=\Phi\left(\gamma_{i}\right)-\Phi_{\text {expected }}\left(\gamma_{i}\right)$,

and is obtained from the very definition of the EPASH. In fact, given a catalog $C$ with $N$ objects, we can quantify the statistical noise in a PASH by:

$\sigma \equiv \sqrt{\frac{1}{m} \sum_{i=1}^{m} \eta\left(\gamma_{i}\right)^{2}}$.

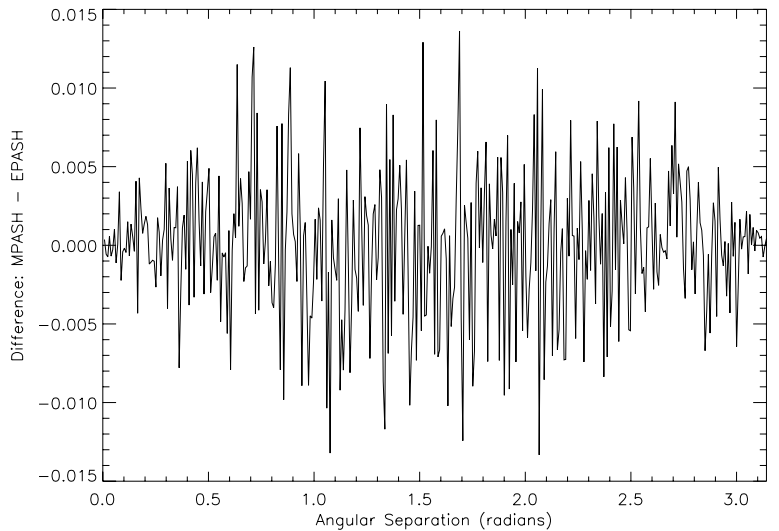

Fig. 6. Difference between PASH and EPASH plotted in Fig. 2 for a full-sky simulated catalog $(K=1)$ with $N=2 \times 10^{3}$ objects and $m=400$ bins; $\sigma=0.00453$. Note that the scale here is different from that of Fig. 5.

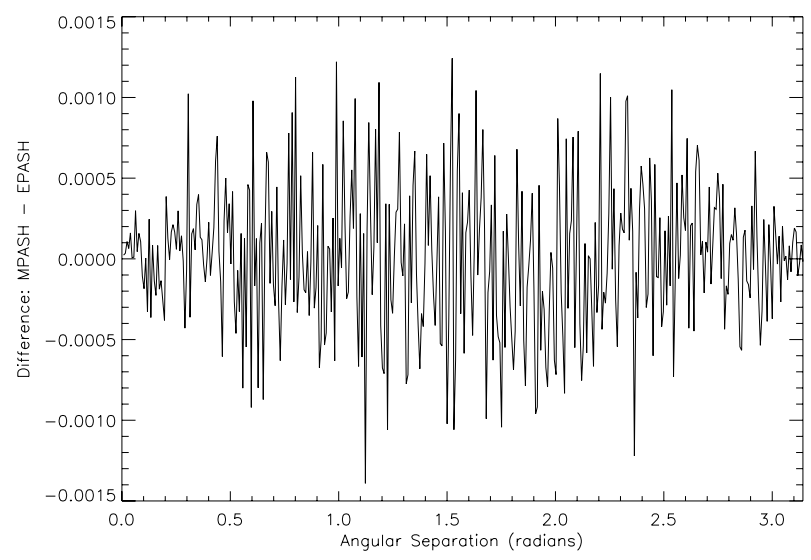

Fig. 7. Difference between MPASH (for a numerical simulation of $K=10^{4}$ full-sky simulated catalogs in $\mathcal{R}^{3}$, with $\langle N\rangle=200$ objects and $m=400$ bins) and EPASH; $\sigma=0.00045$.

For the cases shown in Figs. 5 and 6, we evaluate the statistical noise using Eq. (22) obtaning $\sigma=0.04418$ for $N=200$, and $\sigma=0.00453$ for $N=2 \times 10^{3}$ respectively.

In Fig. 7, we show the difference between the MPASH and the EPASH for a numerical simulation of $K=10^{4}$ fullsky simulated catalogs in $\mathcal{R}^{3}$, with a mean number of $\langle N\rangle \equiv$ $1 / K \sum_{i=1}^{K} N_{i}=200$ objects per catalog. The statistical noise is $\sigma=0.00045$. Fig. 8 shows the difference between a MPASH, obtained from a numerical simulation of $K=10^{4}$ full-sky catalogs with $\langle N\rangle=20$ objects each, and the corresponding EPASH; in this case $\sigma=0.00448$. In Fig. 9, we show the difference between a MPASH and the EPASH, where the MPASH was obtained from a numerical simulation of $K=150$ full-sky simulated catalogs with $\langle N\rangle=100$ objects each, but with $m=600$. In this case $\sigma=0.00926$.

Thus, when we compare the cases shown in Figs. 6 and 8 (equal $m$ and $\sigma$, different values for $N$ and $K$ ) with the cases shown in Figs. 5 and 7 (equal $m$ and $N$, different values for $\sigma$ and $K$ ), we finally arrive at

$\sigma \simeq \frac{0.45 \sqrt{m}}{\langle N\rangle \sqrt{K}}$ 




Fig. 8. Difference between MPASH (for a numerical simulation of $K=10^{4}$ full-sky simulated catalogs in $\mathcal{R}^{3}$, with $\langle N\rangle=20$ objects and $m=400$ bins) and EPASH; $\sigma=0.00448$.

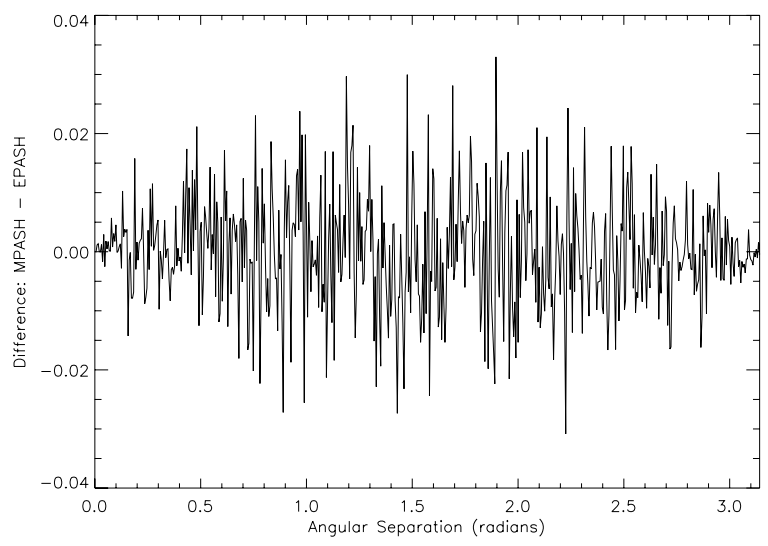

Fig. 9. Difference between MPASH (for a numerical simulation of $K=150$ full-sky simulated catalogs in $\mathcal{R}^{3}$, with $\langle N\rangle=100$ objects and $m=600$ bins) and EPASH; $\sigma=0.00926$.

We can verify this relationship with the aid of the case shown in Fig. 9, in which $m=600$. In fact, using Eq. (23), we obtain $\sigma=0.009$, while calculating the right hand side of Eq. (22) with the values of $\Phi\left(\gamma_{i}\right), i=1, \ldots, 600$, obtained from Fig. 9, results $\sigma=0.00926$.

In Figs. 5 and 6, as well as in Figs. 3 and 4, we notice the absence of correlations between pairs of objects for the exactly isotropic distribution in $\mathcal{R}^{3}$ generated using random numbers for the coordinates. Thus, it is clear from these simulations that as $K \rightarrow \infty$ and/or $N \rightarrow \infty$ the statistical noise measured by $\sigma \rightarrow 0$. The MPASH is related to the statistical noise and this fact is quantified in Eq. (23). Although the statistical noise is inherent to any numerical simulation, it can be controlled in three ways: i) by obtaining catalogs with a large number of elements, in order to produce PASHs with a small noise, since the statistical noise depends on the number of objects; ii) by computing the MPASH to significantly reduce the statistical noise; iii) by choosing a suitable number of bins $m$.

\section{Topological signatures in PASHs}

In any multiply-connected 3 -space $\mathcal{M}_{0}$ whose FP lies inside the ball $\mathcal{B}_{R}$, there might exist images of a given object in a shell of thickness $\Delta R \equiv R-R_{-}$, whenever the horizon scale $R$ is greater than half of the length of the smallest closed geodesic of $\mathcal{M}_{0}$ (Lachièze-Rey \& Luminet 1995; Lehoucq et al. 1996; Fagundes \& Gausmann 1998a,b; Gomero et al. 2000a,b, 2001, 2002). These images give extra contributions to the PASH due to the isometries of $\mathcal{M}_{0}$.

By definition, the probability density for two objects $p, q$ in $C$ be correlated by a translational isometry $g_{i}$ (we represent this correlation by $\left.q=g_{i}(p)\right)$ is

$$
\begin{aligned}
\mathcal{P}^{g_{i}}(\ell) \equiv & \frac{v_{g_{i}}}{n} \int_{\mathcal{B}_{R}} \int_{\mathcal{B}_{R}} \mathrm{~d}^{3} r_{p} \mathrm{~d}^{3} r_{q} \rho\left(\bar{r}_{p}\right) \rho\left(\bar{r}_{q}\right) \\
& \times \delta(d(p, q)-\ell) \delta\left(\bar{r}_{q}-g_{i}\left(\bar{r}_{p}\right)\right),
\end{aligned}
$$

where $\ell$ is the distance variable for objects in $\mathcal{B}_{R}, \ell \in(0,2 R]$; $\mathrm{d}(p, q) \equiv\left|\bar{r}_{p}-\bar{r}_{q}\right|$ is the 3-dimensional Euclidean distance between $p$ and $q$, and the coefficients $v_{i} \equiv v_{g_{i}}$ are the number of images generated by the isometry $g_{i}$. Moreover, $n$ is the density number of objects inside the volume under investigation (in the present case a thin shell), and $N$ is the number of objects in this volume.

Consider the pure translational isometries in $\mathcal{M}_{0}=T^{3}$, with a FP represented by a cube of side $L$. We know that the distances between pairs correlated by Clifford translations are independent of the location of the object (see Gomero et al. 2002), i.e., $\forall p \in \mathcal{B}_{R}$, the distance $\left|g_{i}(p)\right|=\mathrm{d}\left(p, g_{i}(p)\right)=\lambda_{i}$ is independent of $p$. Thus, for this manifold, the translational isometries $g_{i}, i=1,2, \ldots$ are such that for $\lambda_{1}=L$ we obtain $v_{1}=6$, for $\lambda_{2}=\sqrt{2} L$ we obtain $v_{2}=12$, for $\lambda_{3}=\sqrt{3} L$ we obtain $v_{3}=8$, etc.

Let's now apply the above definition to the topological images correlated by the Clifford translation isometries $g_{i}$ and located in a thin shell of thickness $\Delta R$. In this case,

$\rho\left(\bar{r}_{p}\right)=\frac{\Theta\left(R-r_{p}\right) \Theta\left(r_{p}-R_{-}\right)}{\frac{4 \pi}{3}\left[R^{3}-R_{-}^{3}\right]}$.

Using this expression in Eq. (24), and after some calculations, we arrive at

$\mathcal{P}^{g_{i}}(\ell)=P_{i} \Theta\left(R-\frac{\lambda_{i}}{2}\right) \delta\left(\ell-\lambda_{i}\right)$,

which, due to the $\delta$ Dirac-function, appears as an acute peak localized at $\ell=\lambda_{i}$ in any pair distance-separation histogram, and where

$P_{i} \equiv \frac{3 v_{i} \Delta R\left(R+R_{-}\right)^{2}}{8 \lambda_{i} N\left(R^{2}+R R_{-}+R_{-}^{2}\right)}$

gives the height of the peak. We notice that the probability for these isometries to show up in pair separation histograms is proportional to the thickness $\Delta R$ of the shell and inversely proportional to $N$, the number of objects in the shell.

In Euclidean multiply-connected 3-spaces with Clifford translations, the objects located in a thin shell and correlated in distance by the Clifford translational isometries $g_{i}$ (of the covering group $\Gamma$ ) give rise to a definite signature in the PASHs. In fact, for shells of small thickness (like that corresponding to the decoupling era where $\Delta R / R \simeq 0.003$ ), these angular correlations between pairs appear in the MPASHs as small peaks 
and are approximately located at the angular scales

$\gamma_{g_{i}} \simeq 2 \arcsin \left(\frac{\lambda_{i}}{2 R}\right)$

whenever $R \geq \lambda_{\min } / 2$, which is the requirement for the existence of topological images in the observable universe. Thus, it is not difficult to verify that the minimum and the maximum angle ( $\gamma_{g_{i}}^{\min }$ and $\gamma_{g_{i}}^{\max }$, respectively) subtended by two objects located in a thin shell and separated by a isometry distance $\lambda_{i}$ are, respectively:

$$
\begin{aligned}
& \sin \left(\gamma_{g_{i}}^{\min } / 2\right) \simeq \frac{\lambda_{i}}{2 R}, \\
& \sin \left(\gamma_{g_{i}}^{\max } / 2\right) \simeq \frac{\lambda_{i}}{2 R_{-}} .
\end{aligned}
$$

We observe that the probability for angular correlation between objects is a direct consequence of the existence of the $N(N-$ 1)/2 pairs located in the thin shell and correlated by the Clifford translation $\lambda_{i}$ with the probability given in Eq. (27). In fact, the probability density that a pair of objects be correlated by to the isometry $g_{i}$ at an angle $\gamma$, is

$\widehat{\mathcal{P}}^{g_{i}}(\gamma)=P_{i} \frac{\Theta\left(\gamma-\gamma_{g_{i}}^{\min }\right) \Theta\left(\gamma_{g_{i}}^{\max }-\gamma\right)}{\Delta \gamma_{g_{i}}}$,

where $\Delta \gamma_{g_{i}} \equiv \gamma_{g_{i}}^{\max }-\gamma_{g_{i}}^{\min }$. Thus, the topological signatures coming from the isometries $g_{i}$ contribute to the EPASH (i.e. $\Phi_{\text {expected }}^{g_{i}}$, see Eq. 7) as

$\Phi_{\text {expected }}^{g_{i}} \equiv \frac{1}{\delta \gamma} \int \widehat{\mathcal{P}}^{g_{i}}(\gamma) \mathrm{d} \gamma=\frac{P_{i}}{\delta \gamma}$

Therefore, the angular correlations due to Clifford translational isometries appear in the PASHs as triangular peaks with basis $\Delta \gamma_{g_{i}}$ and height $P_{i} / \delta \gamma$.

The effects of the non-translational isometries can also be calculated using Eq. (24). However, in such a case, the integrals are cumbersome to handle, so we prefer to show their effect in MPASHs with plentiful numerical simulations. For this reason, we decided to work with the $G_{6}$ manifold, which does not present Clifford translations in the histograms whenever $R / L \leq \sqrt{2} / 2, L$ being the arista of the cube representing the FP.

\section{Numerical simulations}

We performed numerical simulations aiming to reveal topological signatures due to the isometries of the compact flat manifods $T^{3}, T_{\pi}$ and $G_{6}$ for full sky and for polar-cap simulated catalogs.

It is difficult sometimes to recognize a topological signature in a single PASH due to the presence of statistical noise. As a matter of fact, we found that topological signatures due to Clifford translations appear in PASHs as small triangular peaks whose heights are inversely proportional to the number of objects in a given catalog (see Eq. (26)). We saw that it is convenient to divide a catalog of $N$ objects in $K$ sub-catalogs of $\langle N\rangle=N / K$ objects, where the objects in a sub-catalog share common physical properties, and then perform the MPASH of the $K$ resulting PASHs. By doing this, we magnify in two ways the presence of the topological signatures: reducing the number of objects per catalog, $N \rightarrow\langle N\rangle$, the height of the peak increases by a factor $N /\langle N\rangle=K$; and obtaining $K$ comparable catalogs we can perform the MPASH to reduce the noise by a factor $1 / \sqrt{K}$. The net result is to increase the signal-to-noise ratio (SNR) of the topological signatures by a factor $\sqrt{K}$. This explains why the strategy of dividing in sub-catalogs the original catalog with a large $N$, and performing the MPASH works better than considering just the PASH of the original catalog. For 3D catalogs, that is, when the 3 spatial coordinates of the objects are provided, the Collecting Correlated Pairs method (Uzan et al. 1999) is suitable to enhance the SNR for the signatures originated by the holonomies of the 3-space $\mathcal{M}_{k}$.

In the simulations presented in this section we assume that the FP is a cube of side $L=1$, and the origin of coordinates is in the center of this cube. Moreover, for $T^{3}$ and $T_{\pi}$, we consider $R \equiv R_{+}=1, R_{-}=0.997$, i.e. $\Delta R=0.003$. The case of $G_{6}$ is doubly special: firstly, in order to avoid the presence of Clifford translations appearing at $\lambda=\sqrt{2} / 2$, we assume $R=0.7$; secondly, because the non-translational isometries have a very small signature in the MPASH, we assume $R_{-}=0.69$, i.e. $\Delta R=0.01$.

In order to normalize and compare the results appearing in the different MPASHs shown below, we fix the values of $m$, and consequently that of $\delta \gamma$, in all of them. Thus, we consider that the interval $[0, \pi]$ is divided in $m=400$ bins, each one of width $\delta \gamma=\pi / m=0.00785$.

In general, to clearly reveal the signatures in the MPASH, a good SNR is of the order of 10; in particular, regarding Clifford translational isometries, this implies that the number of catalogs should be such that

$\frac{P_{i}}{\delta \gamma} \gtrsim 10 \sigma$.

Thus, using the expression for $P_{i}$, Eq. (27), and the numerical values for the parameters involved, we obtain that, for $K \gtrsim$ $10^{4}$, the topological signatures due to Clifford translations are clearly revealed in the MPASHs.

\subsection{MPASHs for full-sky maps}

In this section, we show how to generate full-sky catalogs in order to construct the MPASHs to reveal topological signatures in the angular distribution of cosmic objects. We assume in all the simulations presented here that the FP is a cube of side $L=$ 1 , centered at the origin of coordinates. To construct a PASH through a numerical simulation, we first randomly generate a set of three Euclidean coordinates $x, y, z \in[-0.5,0.5]$ for $N_{\text {seeds }}$ objects located inside the FP.

In order to be sure that the distribution generated in this way is uniform, we test the randomness of the simulation using the Pair Separation Histograms for the simply-connected case $\mathcal{R}^{3}$, according to the theoretical relationship expected for such a case given by Bernui \& Teixeira (1999). After that, these $N_{\text {seeds }}$ objects are mapped to the whole ball $\mathcal{B}_{R}$ via the translational isometries $g_{i} \rightarrow \lambda_{i}=1, \sqrt{2}, \sqrt{3}, \ldots$ of $T^{3}$, giving rise to $N_{T}$ objects (including the $N_{\text {seeds }}$ objects) in $\mathcal{B}_{R}$. Then, we select the 


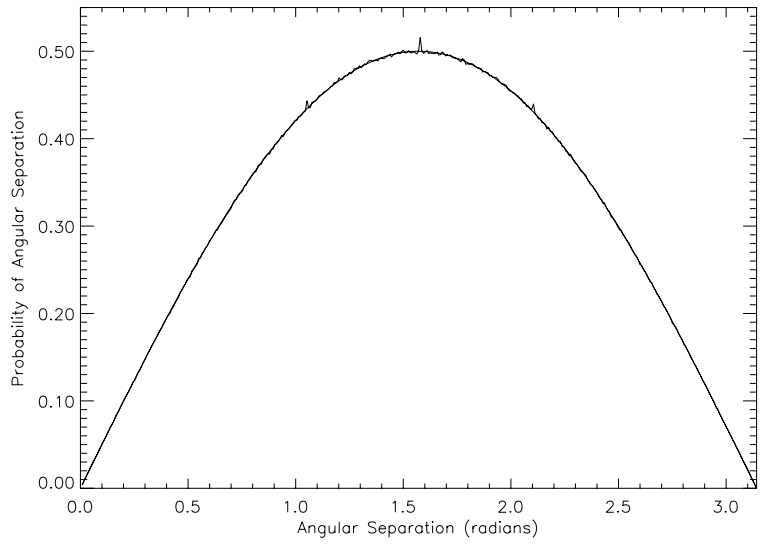

Fig. 10. MPASH together with EPASH for $K=10^{4}$ full-sky simulated catalogs in $T^{3}$ (the FP is a cube of side 1 ); $\langle N\rangle \simeq 100$ objects.

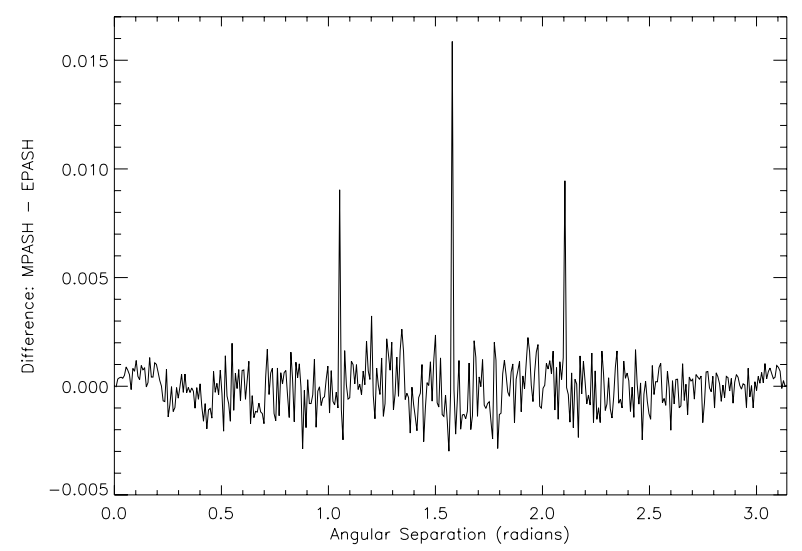

Fig. 11. Difference between MPASH and EPASH corresponding to Fig. 10.

$N$ objects located inside the thin shell, i.e. those objects with radial distance satisfying $R_{-} \leq r_{i} \leq R, i=1, \ldots, N$. We project them on the involving sphere $\mathcal{S}_{R}$ and calculate all the angular distances between the $N(N-1) / 2$ pairs of objects.

We then count how many of these angular distances are in the interval $J_{i}, i=1,2, \ldots, 400$. Finally, we normalize the PASH and plot the number of distances versus the angular distance $\gamma$. For the simulations showed in Figs. 10 and 11, where we consider the case $\mathcal{M}_{0}=T^{3}$, we use $R=1, R_{-}=0.997$. In this case, due to the ratio between the volumes of $\mathcal{B}_{R}$

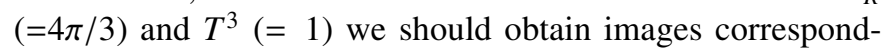
ing to the first three Clifford translations: $\lambda_{1}=1, \lambda_{2}=\sqrt{2}$, and $\lambda_{3}=\sqrt{3}$. As a consequence of this, the MPASH presents angular correlations that are approximately located at $\gamma_{g_{i}}^{\min } \simeq$ $1.047,1.571,2.094$ radians, respectively, with the corresponding peak heights $P_{i} / \delta \gamma \sim 1.14 \times 10^{-2}, 1.6 \times 10^{-2}$, and $0.9 \times 10^{-2}$.

For the simulation showed in Fig. 12, where we study the case $\mathcal{M}_{0}=T_{\pi}$, we also used $R=1, R_{-}=0.997$. However, in this case, the Clifford translations contributing to the topological angular correlations studied in the previous section are just two: $\lambda_{1}=1, \lambda_{2}=\sqrt{2}$, which generate only the images $v_{1}=$ $4, v_{2}=4$, respectively. According to this, the heights of the 2 peaks appearing in Fig. 12 are $P_{i} / \delta \gamma \sim 7.6 \times 10^{-3}, 5.4 \times 10^{-3}$, and are approximately located at $\gamma_{g_{i}}^{\min } \simeq 1.047,1.571$ radians, for $i=1,2$ respectively. In fact, as we can observe in Fig. 13,

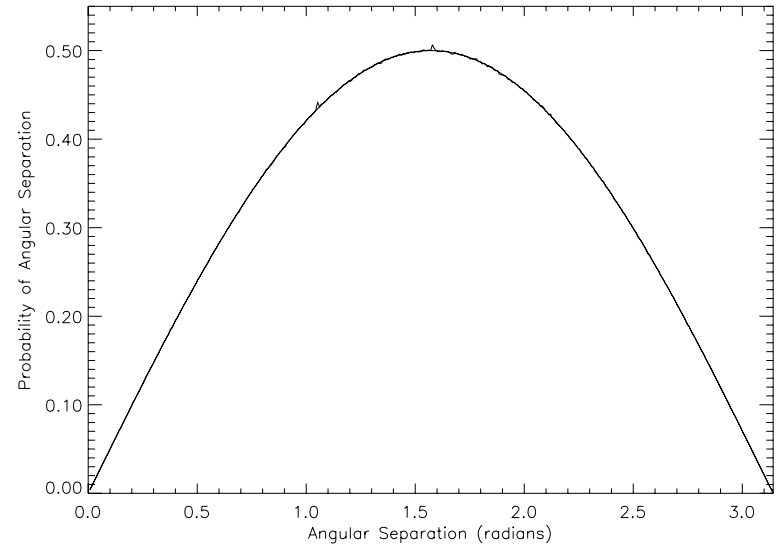

Fig. 12. MPASH together with EPASH for $K=2 \times 10^{4}$ full-sky simulated catalogs in $T_{\pi}$ (the FP is a cube of side 1 ), $\langle N\rangle \simeq 100$ objects.

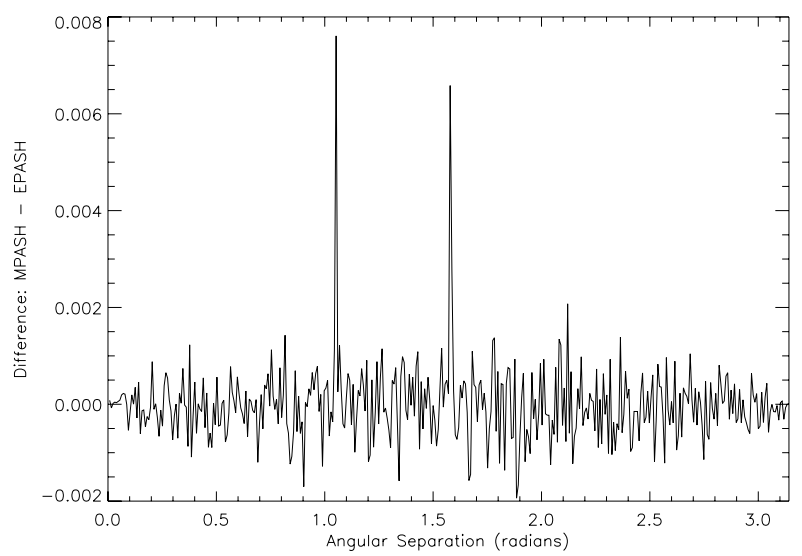

Fig. 13. Difference between MPASH and EPASH corresponding to Fig. 12.

there is no peak at $\gamma_{g_{i}}^{\min } \simeq 2.094$ because there is no translational isometry at $\lambda=\sqrt{3}$ in $T_{\pi}$.

In Fig. 14 we present the MPASH and the EPASH for the case of the 3-space $\mathcal{M}_{0}=G_{6}$, and in Fig. 15 we show the corresponding difference between them. In this case, we assumed again that the FP is a cube of side $1, R=0.7, R_{-}=0.69$, i.e. $\Delta R=0.01,\langle N\rangle \simeq 20$ objects, and $K=4 \times 10^{5}$.

Comparing Figs. 11 and 13 with Fig. 15 we observe that the heights of the peaks are much smaller in the case of screw-motion isometries than in the case of Clifford translation isometries. However, our simulations show that with a suitable number of catalogs, the screw-motions, as well as the pure translational isometries, are revealed in the MPASHs as small peaks.

\subsection{MPASHs for polar-cap maps}

We present numerical simulations in which the objects are located in a thin spherical shell of thickness $\Delta R$; the radial distance of the object to the origin of coordinates is $R \leq r \leq R_{-}$, $\theta_{0}=70^{\circ}$. We performed these calculations for both $T^{3}$ and $T_{\pi}$ 3-spaces.

A noticeable fact, shown in Figs. 11, 13, and 15 corresponding to the numerical simulations done in the precedent 


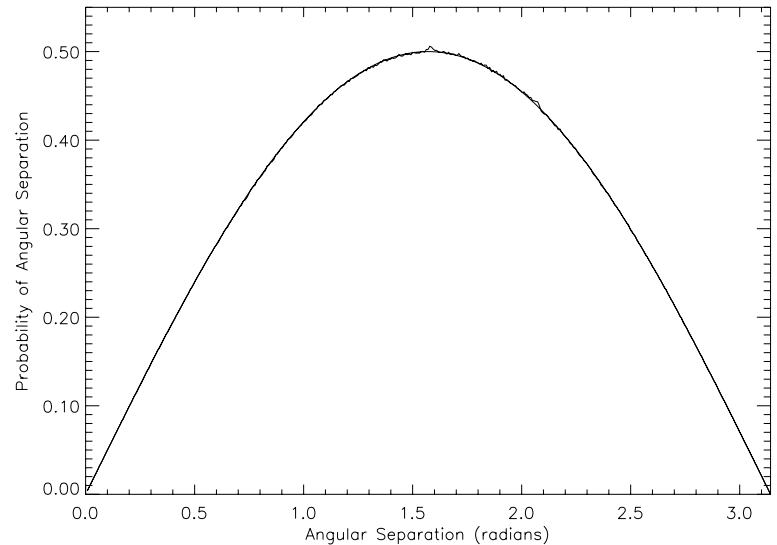

Fig. 14. MPASH together with EPASH for $K=4 \times 10^{5}$ full-sky simulated catalogs with objects in $G_{6}$ (the FP is a cube of side 1 ), $R=0.7, R_{-}=0.69$ (i.e. $\Delta R=0.01$ ), $\langle N\rangle \simeq 20$ objects.

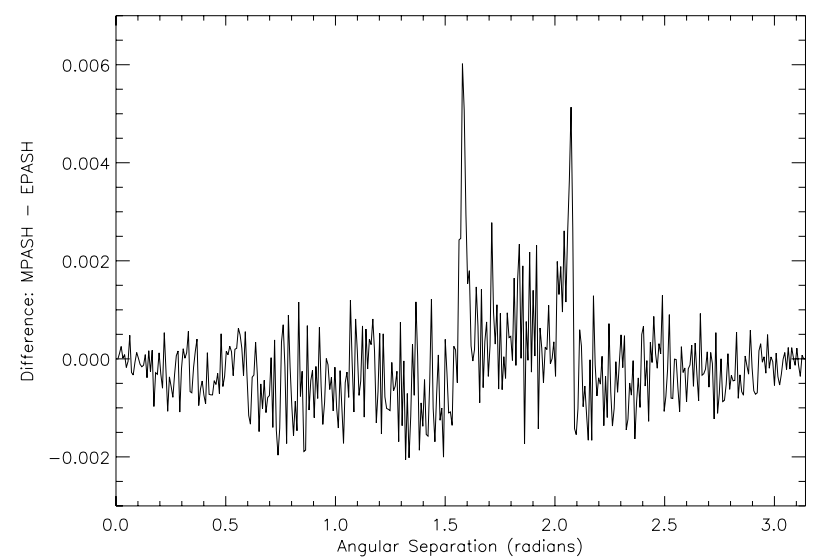

Fig. 15. Difference between MPASH and EPASH corresponding to Fig. 14.

sub-section, is that full-sky catalogs produce small distinguishable peaks in the MPASHs when the isometries are Clifford translations (which appears only in Euclidean and spherical geometries; for details see, e.g., Gomero et al. 2000a,b, 2001, 2002), and even smaller signatures for the non-translational isometries.

For completeness we perform now numerical simulations in order to test whether these results are valid for partial-sky catalogs, namely for polar-cap catalogs, and we do this for the cases when the 3 -space is $T^{3}$ and $T_{\pi}$. Observing Figs. 17 and 18 , we notice that the topological information does not disappear when we consider a suitable polar-cap catalog instead of a full-sky catalog.

Regarding our simulations, we remark that the values assumed for $R$ and $L$ means that the observable Universe includes a small number of multiple images, generated by the isometries of the manifold, for each seed-object present in the FP. This assumption is within the limits imposed by CMBR to the scale of the Small Universe considering a $T^{3}$ manifold (e.g. Stevens et al. 1993; Starobinsky 1993; de Oliveira-Costa \& Smoot 1995; de Oliveira-Costa et al. 1996).

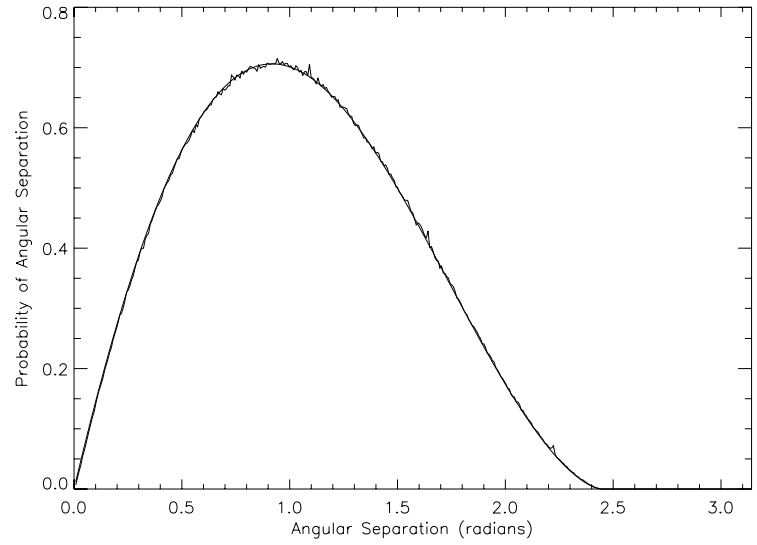

Fig. 16. MPASH together with EPASH for a polar-cap catalog in $T^{3}$, with $\theta_{0}=70^{\circ},\langle N\rangle \simeq 40$ objects, $K=4 \times 10^{4}$.

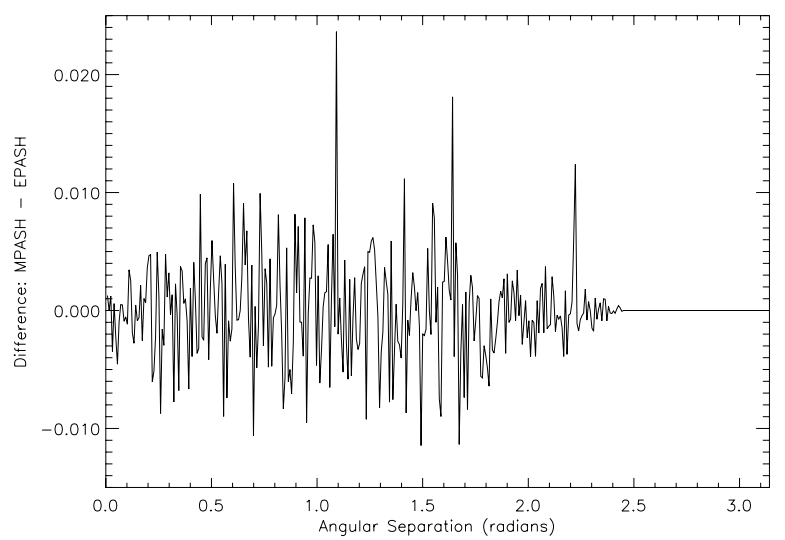

Fig. 17. Difference between MPASH and EPASH plotted in Fig. 16.

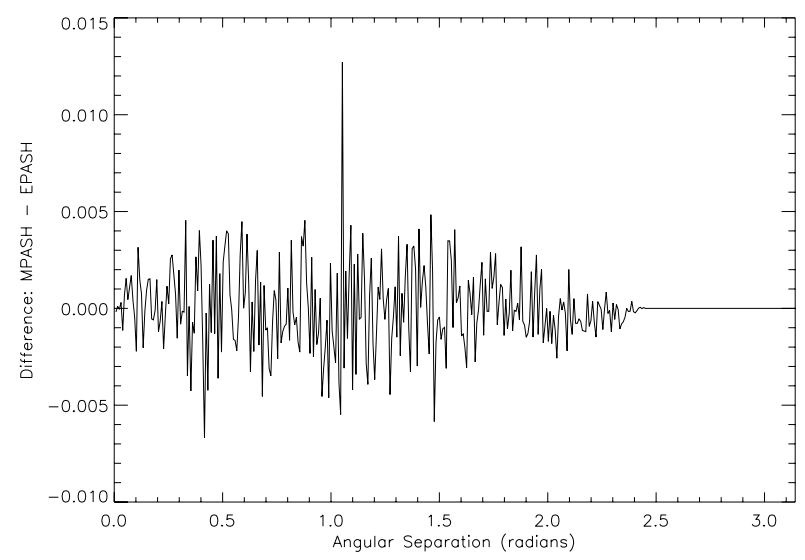

Fig. 18. Difference between MPASH and EPASH obtained for a polarcap catalog (with $\theta_{0}=70^{\circ}$ ) for the case where the 3-space is $T_{\pi}$; $\langle N\rangle \simeq 40$ objects and $K=4 \times 10^{4}$. The axis of symmetry of the polar-cap coincides with the axis of the screw-motion isometry.

\section{Discussion and conclusions}

We presented a method to study the angular distribution of cosmic objects. It has the great advantage of being independent of cosmological models or parameters. Through the analyses of the PASHs, we investigated the effect that different topological properties, termed Euclidean isometries, produce in the 
angular distributions of objects. We performed these analyses in full-sky as well as in polar-cap catalogs.

We concentrate on the analysis of objects located in a thin spherical shell, considering only their angular positions in the celestial sphere in order to explore CMBR data. We have considered four different cases regarding the 3-space where the shell is embedded: the simply-connected $\mathcal{R}^{3}$, and the three topologically different multiply-connected flat 3-spaces: $T^{3}$, $T_{\pi}$, and $G_{6}$.

In the simply-connected case, topological angular correlations are clearly absent. This is evinced by plotting the difference between the MPASH and the EPASH, where only statistical fluctuations appear, as seen in Figs. 5 to 9, independently of the number of simulated catalogs used to produce the MPASH. For the multiply-connected 3-spaces analyzed, angular correlations due to the isometries involved in each specific case indeed appear. Thus, the topological imprints corresponding to translational isometries manifest themselves as small and independent peaks, i.e., one peak for each isometry. On the other hand, the signatures corresponding to the nontranslational isometries (which is the case for the $G_{6}$ manifold) appear as a less intense but extended angular correlation: from $\gamma_{g_{2}}^{\min } \simeq 1.571$ radians to $\gamma_{g_{3}}^{\min } \simeq 2.094$, as can be seen in Fig. 15 .

It is also important to notice that the topological information of multiply connected 3-spaces does not disappear when we consider a suitable polar-cap catalog instead of a full-sky catalog, that is, provided that such polar-cap is sufficiently large and conveniently oriented in the sky to map multiple images. This fact is clearly observed in Figs. 17 and 18. This is a very useful result because in general astronomical data are presented in catalogs covering only partial regions of the sky. In order to improve the SNR and reveal small topological imprints in the angular distribution of cosmic objects, the strategy is to divide the original catalog in several sub-catalogs and then perform the MPASH. It could be also useful to divide the fullsky data in a set of antipodal polar-caps and compare them to similar sky patches in a statistically isotropic Universe (Bernui et al. 2004).

We have shown that a topological signature, no matter how small it is, can always be revealed whenever one achieves a suitable SNR in the difference between the MPASH and the EPASH. As shown in our simulations, the small topological signatures of $T^{3}$ and $T_{\pi}$ are clearly revealed when averaging $K=4 \times 10^{4}$ catalogs with $\langle N\rangle \simeq 40$ objects each, from an original catalog of $1.6 \times 10^{6}$ cosmic objects. For $G_{6}$, the number of cosmic objects should be five times larger. For the case of CMBR maps which are always contaminated by Galactic foregrounds, the polar-cap analysis described in this paper would be useful to reveal possible angular correlations in these data sets.

Acknowledgements. We thank A.F.F. Teixeira, G.I. Gomero, M.J. Rebouças, and C.A. Wuensche for many fruitful discussions. A.B. thanks CNPq (PCI/DTI7B fellowship) and TWAS for the partial financial support, and the hospitality of the Centro Brasileiro de Pesquisas Físicas visited under the TWAS-Associateship Scheme at Centres of Excellence in the South. T.V. was partially supported by CNPq grant 302266/88-7-FA and FAPESP grant 00/06770-2. We also thank the anonymous referee for the valuable comments on the manuscript.

\section{References}

Bernui, A., Gomero, G. I., Rebouças, M. J., \& Teixeira, A. F. F. 1998, Phys. Rev. D, 57, 4699

Bernui, A., \& Teixeira, A. F. F. 1999 [arXiv:astro-ph/9904180]

Bernui, A., Villela, T., \& Ferreira, I. 2004, Int. J. Mod. Phys. D, 13, 1189

de Oliveira-Costa, A., \& Smoot, G. F. 1995, ApJ, 448, 477

de Oliveira-Costa, A., Smoot, G. F., \& Starobinsky, A. A. 1996, ApJ, 468,457

Ellis, G. F. R., \& Schreiber, G. 1986, Phys. Lett. A, 115, 97

Fagundes, H. V., \& Gausmann, E. 1998 [arXiv: astro-ph/9811368]

Fagundes, H. V., \& Gausmann, E. 1998, Phys. Lett. A, 238, 235

Friedmann, A. 1922, Z. Phys., 10, 377

Gomero, G. I., Rebouças, M. J., \& Teixeira, A. F. F. 2000a, Phys. Lett. A, 275, 355

Gomero, G. I., Rebouças, M. J., \& Teixeira, A. F. F. 2000b, Int. J. Mod. Phys. D, 9, 687

Gomero, G. I., Rebouças, M. J., \& Teixeira, A. F. F. 2001, CQG, 18, 1885

Gomero, G. I., Teixeira, A. F. F., Rebouças, M. J., \& Bernui, A. 2002, Int. J. Mod. Phys. D, 11, 869

Lachièze-Rey, M., \& Luminet, J.-P. 1995, Phys. Rep., 254, 135

Lehoucq, R., Lachièze-Rey, M., \& Luminet, J.-P. 1996, A\&A, 313, 339

Lemaître, G. 1927, Ann. Soc. Sci. Bruxelles, 47, 29

Starobinsky, A. A. 1993, JETP Lett., 57, 622

Stevens, D., Scott, D., \& Silk, J. 1993, Phys. Rev. Lett., 71, 20

Teixeira, A. F. F. 2003 [arXiv:physics/0312013]

Uzan, J.-Ph., Lehoucq, R., \& Luminet, J.-P. 1999, A\&A, 351, 766

Wolf, J. A. 1984, Spaces of Constant Curvature (Delaware: Publish or Perish Inc.) 